REVIEW ARTICLE

\title{
Genetic Basis of Male Infertility
}

\author{
*MR Begum ${ }^{1}$, M Ehsan ${ }^{2}$ \\ *1Prof. Dr. Mosammat Rashida Begum, Professor and Head, Dept. of Obs \& Gyn \\ Anwer Khan Modern Medical College, Dhaka \\ ${ }^{2}$ Dr. Mariya Ehsan, Medical Officer, Infertility Care and Research Centre (ICRC), Dhaka \\ *Corresponding author
}

\begin{abstract}
Infertility is a couple's problem. Almost $50 \%$ case males are responsible for infertility. Most common cause is oligospermia and azoospermia and approximately 5\% to $15 \%$ of men with azoospermia and severe oligospermia may have a chromosomal abnormality. Men with significant spermatogenic compromise are the candidates of intracytoplasmic sperm injection (ICSI). Raised FSH level above 9 is an indication of spermatogenic compromise. So, medical treatment for these patients is waste of time and money. Early attempt of assisted reproduction is ideal to avoid the crisis of total spermatogenic failure in near future. But before going for ICSI genetic testing if possible and proper counseling about possibilities of transmission of genetic disease to offspring is necessary.
\end{abstract}

Key words: Male Infertility, Genetic Basis

\section{Introduction}

Infertility is a couple's problem. Male factors have been estimated to contribute to infertility in $25 \%$ to $50 \%$ of infertile couples ${ }^{1}$. Male infertility is not one disorder but is a syndrome that results from many congenital and acquired illnesses. Main causes of infertility in men are oligospermia and azoospermia. Approximately $5 \%$ to $15 \%$ of men with azoospermia or severe oligospermia may have a chromosomal abnormality $^{2}$. Chromosomal abnormalities can be numerical or structural and involve the sex and or autosomal chromosomes. Numerical abnormalities involve the gain/ loss of a complete chromosome and structural abnormalities results from mistakes in the repair of chromosome breaks or from malfunction of the recombination system. As so many men with significant compromise of the spermatogenic axis may be candidates for intracytoplasmic sperm injection (ICSI) it is of great importance to identify a genetic basis if possible for proper counseling of patients about possibilities of transmission of abnormal gene to male offspring. Couple after knowing everything can make the best decision about their reproduction.

\section{Responsible chromosomal abnormalities are:}

\section{Numerical abnormalities}

i). Klienfelter's Syndrome: ( $47 \mathrm{XXY}$ ) :This syndrome is found in approximately 1 in 500 males and in the result of non-disjunction of the $\mathrm{X}$ chromosome during meiosis. This is the most sex chromosomal abnormality detected in men with non-obstructive azoospermia (NOA). Usual features are raised serum levels of gonadotropins, reduced serum levels of testosterone, and reduced testicular volume, libido, hypoandrogenization and almost total atrophy of seminiferous tubules. Most men are azoospermic and some are oligospermic. In 50\% cases of Klinfelter's syndrome sperm found upon testicular sperm extraction (TESE). The sperm can serve as the source of the male gamete to establish pregnancy with $\mathrm{ICSI}^{3}$.

ii). XX males a variant of Klinefelter's syndrome with small testes and associated with infertility. It occurs with translocated portion of the Y chromosome carrying the SRY gene. To date $46 \mathrm{XX}$ males have been found to possess no level of spermatogenesis within harvested 
testicular tissue. So a karyotype $46 \mathrm{XX}$ male syndrome is completely prognostic and absolutely predicts that TESE will not be successful. c) $47 \mathrm{XYY}$ is another problem occur among 1 per 1000 men. Spermatogenesis ranges from normal to severely impaired. iii). Isodentric $\mathrm{Y}$ chromosome and Ring $\mathrm{Y}$ chromosome are responsible for non-obstructive azoospermia (NOA).

\section{Structural abnormalities}

i). Microdeletion of $Y$ chromosome: Microdeletion of the section of the $\mathrm{Y}$ chromosome may be detected in $10 \%$ to $15 \%$ of azoospermic men and $3 \%$ to $10 \%$ of oligospermic men with normal karyotype $(5,6)$. These are too small that cannot be detected by karyotyping but can be identified with sequenced-tagged site probes along entire length of the Y chromosome. Most deletions associated with azoospermia or oligospermia occur in non-overlapping regions of the long arm of the $\mathrm{Y}$ chromosome (Yq11) designated as $\mathrm{AZFa}, \mathrm{AZFb}, \mathrm{AZFc}$ that contain multiple genes important for spermatogenesis. The specific location of any $\mathrm{Y}$ chromosome microdeletion may determine the extent to which spermatogenesis is affected.

Microdeletion of the AZFa region is found less than $1 \%$ of men with NOA. These patients are highly unlikely to have sperm found upon TESE, as it is associated with germinal aplasia. Therefore TESE is not required and treatment by ICSI is not possible. Deletion of AZFb region (e.g in the RNA binding motif) is associated with azoospermia and maturational arrest. The prognosis for sperm retrieval in men with deletions involving the entire length of $\mathrm{AZFb}$ region is generally very poor, even with multiple biopsies?

An AZFc microdeletion occur 1 in 4000 men and is the common molecular cause of NOA. DAZ (deleted azoopsrmic gene is located in the $A Z F c$ region). Thirteen percent of men with NOA will be AZFc microdeleted. Approximately $70 \%$ of men with an AZFc microdeletion posse's sperm either in ejaculate (but severe oligospermia) or in testicular tissue in sufficient numbers to allow sperm recovery from a testis biopsy. It is also associated with varying stages of maturational arrest. About 6\% of men with severe oligospermia $<5$ million /cc are midrodeleted. Sons of affected individuals will inherit the microdeletion and may be similarly infertile6. So ICSI in severe oligospermia and NOA should be offered after testing and counseling. ii). Balanced translocations with no net gain/loss of chromosomal material involving one of the sex chromosomes resulting in reduced sperm production. iii). Unbalanced translocations with net gain/loss of chromosomal material associated with spermatogenic abnormalities e.g. chromosomally unbalanced haploid sperm. iv). Robertsonian translocations involving 2 acrocentric chromosomes that fuse near the centromere region with the loss of short arms causing azoospermia, oligospermia or sperm maturational arrest at the secondary spermatocyte stage. These translocations can result in an unbalanced karyotype in the foetus resulting in a miscarriage, and nonviable foetus. v). Disruption of the SOX9 gene is associated with sex reversal and gonadal dysgenesis in male (46 XY female) results in NOA. vi). Mutations in the GnRH receptor gene on long arm of chromosome 4 cause hypogonadism. Mutation in LH receptor gene can result low testosterone synthesis, spermatogenic arrest and male pseudoharmaphroditism and mutations of the FSH gene causes oligospermia. vii). Mutations in any of the genes coding for enzymes involved in steroid biosynthesis can lead to hypogonadism and male pseudoharmophroditism. viii). Mutation of the androgen receptor gene (Xq11-q12) causes testicular feminization and hypospadiasis and Kennedy's disease resulting in testicular atrophy and reduced fertility. ix). Deletion of 15 q11.2q13.2 on paternal chromosome (Long arm of 15) results in Prader Willi syndrome associated with hypogonadism. $\mathrm{x}$ ). Mutation of the DMPK gene of chromosome 19q13.3 causes myotonic dystrophy resulting in testicular tubular atrophy. xi). Mutation of FDG1 gene (chromosome Xp11.21) associated with cryptorchidism, ALDP gene (chromosome Xq28) associated with adrenomyeloneuropathy and azoospermia or oligospermia and AIRE gene (chromosome 21q22.3) associated with hypogonadism. xii). Mutation of the cystic fibrosis transmambrane conductance regulator gene (CFTR) on chromosome 7 is associated with cystic fibrosis 
and congenital bilateral absence of the Vas deference (CBAVD) causes obstructive azoospermia. CBAVD is found in approximately $1 \%$ in infertile males and $10 \%$ to $15 \%$ of azoospermic men and one of the most common diagnosis in-patients with obstructive azoospermia. The vas is not palpable and epididymal remnants are distended. The capute of the epididymis is always present. Palpation is therefore the key to diagnosis 8,9 .

Azoospermia and severe oligospermia mostly related to chromosomal defect, which is not correctable. There is a notion that the FSH must be exceptionally high that is about 2-3 times the upper limit for spermatogenic failure to be conclusively diagnosed. This is not correct. The value of FSH in men with perfectly adequate spermatogenesis is usually in the lower aspect of the reference range for any particular assay. When spermatogenic capability is compromised the pituitary compensates with an increase secretion of FSH. Therefore in the face of azoospermia without the evidence of obstruction on history or physical examination and in severe oligospermia a FSH value above the lower limits of the reference range indicates spermatogenic compromise. In those cases no medical treatment is recommended, as genetic problem is most likely. Karyotyping, polymerase chain reaction (PCR) and fluorescent in situ hybridization (FISH) technique can make diagnosis. Where genetic factor exists, early attempt of assisted reproduction is ideal to avoid the crisis of total spermatogenic failure in near future.

\section{But things should be kept in mind that}

- Before any operative intervention or use of sperm for ICSI an Y chromosome microdeletion assay and karyotype should be performed in any male with severe oligospermia or NOA.

0 Congenital bilateral absence of the vas deference is associated with mutations within CF gene in $70 \%$ to $80 \%$ of men. So all men who present with vasal agenesis require a $\mathrm{CF}$ mutation analysis prior to intervention.

0 Proper genetic testing and counseling is necessary prior to ICSI with own sperm.

- Patients should be well informed about the genetic basis for their reproductive failure.

\section{Conclusion}

Treatment of male infertility is a challenge for fertility specialist. Specially when patients suffer from severe oligospermia and azoospermia. Although $90 \%$ oligospermia is idiopathic, result of empirical medical treatment, nutritional supplementation, life style changes is not very satisfactory. Correction of specific cause sometimes improves the situation. In obstructive azoospermia if numerous spermatozoa present (at the level of normospermic men) behind the obstruction, vasovasostomy may improve the situation provided female partner is young and fertile. For other azoospermic and severe oligospermic cases when there is testicular tissue damage due to any cause and genetic factors are responsible ICSI is the only option of treatment after proper counseling.

\section{References}

1. Jones HW Jr, Toner JP. The infertile couple. N Engl J Med 1993; 329: 1710.

2. De Braekeleer M. Dao TN. Cytogenetic studies in male infertility: a review. Hum Reprod 1991; 6: 245-50.

3. Rosenlend B Hreinsson JG, Hovatta O. Birth of a healthy male after frozen thawed blastocyst transfer following intracytoplasmic injection of frozen thawed testicular spermatozoa from a man with nonmosaic Klinefelter's syndrome. J Assist Reprod Genet 2002; 19: 149-51.

4. Skakkebaek NE, Hulten M, Jacobsen $P$, et al. Quantification of human seminiferous epithelium 11. Histologic studies in eight 47, XYY men. J Reprod Fertil 1973; 32: 391.

5. Pryor JL, Kent-First $\mathrm{M}$, Muallem A, et al. Microdeletions in the Y chromosome of infertile men. N Engl J Med 1997; 336: 534-9.

6. Kent-First MG, Kol S, Muallem A, et al. The incidence and possible relevance of $\mathrm{Y}$-linked microdeletions in babies born after intracytoplasmic sperm injection and their infertile fathers. Mol Hum Reprod 1996;2:943-50.

7. Brandell RA, Mielnik A, Liotta D, et al. AZFb deletions predict the absence of spermatozoa with testicular sperm extraction: preliminary report of a prognostic genetic test. Hum Reprod 1998; 13: 2812-5.

8. Chillon M, Casals T, Mercier B, et al. Mutations in the cystic fibrosis gene in patients with congenital absence of the vas deference. N Engl J Med 1995; 332: 1475-80.

9. Dumur V, Gervais R, Rigot JM, et al. Congenital bilateral absence of the vas deference (CBAVD) and cystic fibrosis transmembrane regulator (CFTR): correlation between genotype and phenotype. Hum genet 1996; 97: 7-10. 